\title{
Images of Health Among Hospital Patients in Jordan A qualitative Study
}

\author{
Noordeen Shoqirat \\ Head of Fundamental and Adult Health Nursing at Faculty of Nursing, Mutah \\ University. Jordan \\ E-mail: noordeen@mutah.edu.jo
}

\begin{abstract}
The purpose of this study was to examine hospital patients' understanding of health and its cultural constructions in Jordan. Four focus group discussions were carried out with hospital patients. The findings showed three images of health among patients in Jordan with different complexity levels. This included health as the freedom from illness, health as being in good social and psychological wellbeing, health as being physically, psychologically, socially and economically satisfied. Gender related conceptualisation of health was associated with the role of men and women in the society. Hospital patients' understanding of health is diverse, multidimensional, and affected by their gender. Therefore, hospital nurses are urged to incorporate patients' conceptualisations of health and factors involved into the framework of the patient's daily care plan and health promotion activity. The study limitations and recommendations for practice and future research are also outlined.
\end{abstract}

Keywords: focus group discussions, health, hospital patients, culture, Jordan, nursing.

\section{Introduction}

The concept of health has increasingly been the focus of the literature and widely linked to the nurses' role in health promotion, specific cultures and health service development. [1,3] It is argued that, in order to understand health promotion, having an idea of what to promote is vital. [4,5] Likewise, hospital nurses are unlikely to improve health and to bring about change unless they have adequate 
understanding of the patients' conceptualisation of health and its determinants.[6,7] Thus, if people's health is to be promoted effectively, the concept of health needs to be explored culturally.

Against this background, whilst there are many international studies about hospital nurses' role in promoting patients' health, $[8,12]$ little evidence is available about how patients understand health from their own perspective. Previous studies have tended to be small-scale research, focusing on only one gender or age group, and their conclusions were not verified by replication. [6, 13,14] The lack of data on patients' health conceptualisations might create a mismatch between the activity in health promotion, and their actual needs and expectations.

In Jordan, although the health care services have been developed over the last decade, it focuses often on curing illnesses as opposed to promoting patients' health. $[15,16]$ To date no study has examined how hospital patients understand and construct health during hospitalisation. In light of the current existing evidence, offering congruent health care that could decrease the fear of health services, maximise the respect between patients and nurses is problematic. $[17,18]$

Subsequently establishing culturally congruent health care as well as health promotion activities will not only be lacking empirical guidance, [12, 19] but also their cultural applicability will be in doubt. [20] The current study examines qualitatiavly hospital patients' understanding of health and its cultural constructions in Jordan. Once hospital patients' understanding of health is identified and verified by the literature, hospital nurses, as health promoters, can incorporate them into the framework of the patient's daily care plan, and thus achieve a higher level of compliance with the prescribed treatment. [7, 13].

\section{Background}

When health related literature is reviewed it becomes obvious that the concept of health is still one of the most frequently reported concepts. Health has not only been associated with people's health behaviour $[6,21]$ but also with the population's mortality, morbidity, life satisfaction, happiness, health policy, sexual health and economy. [22,24] The concept of health however is contested and has diverse and sometimes conflicting meanings that are both socially and culturally constructed. Health was defined by WHO as:

"The state of complete physical, mental, and social wellbeing and not only the absence of disease and infirmity". [25] Other scholars[4, 7, 26] propose that if health is to fit different contexts, it needs to be seen as an ability to adapt continually to changing demands, expectations and stimuli. In line with this, it is suggested health actualisation involves the ongoing integration of mind, body and environment. [27-28] 
The theoretical framework of cultural explanatory models (CEMs) explains health-related behaviour by focusing on socio-cultural context, knowledge and attitudes around patients' understanding of health. It is not surprising therefore that nurses are urged to examine how health needs of individuals are specifically expressed and met in a certain culture rather than exclusively practising within a context of a pre-established model of care. $[17,29,30]$ Such models were largely generated and tested within the western paradigm of health. [4, 28] On this basis, there are concerns that the existing models might not fit with other international health care systems.

Hospital nurses are likely to be exposed to different cultures and backgrounds. Arguably, if nurses are to productively offer health promotion activities, they should understand these differing cultural norms. [13,14] Evidence also suggests that when health care providers work with patients' beliefs rather than against them the outcomes are more successful. [6, 29] Consequently, the increased understanding of the subjective experience of health might result in a better informed, and likely a more successful health promotion strategy. That means, research into the meaning of health is not only desirable - rather a prerequisite for establishing health promotion within a specific cultural environment. [7, 27]

However, this is not the case. The review of literature has revealed that the concept of health has been marginalised by international research with particular reference to nursing literature. The existing evidence has not been well verified by recent studies. [31,32] However, the literature showed that health is seen as the absence of disease, physical fitness, functional capacity, strength and psychological well-being. In a qualitative research it was found that elderly women view health differently and their interpretations of the meaning of health have been addressed by a number of themes. [13] These include, attending religious activities particularly in the church. Likewise, women have highlighted the importance of praying regularly to God for both illness and wellness status. These findings are similar to those reported by Hispanic researchers. [14] Their qualitative findings showed that elderly Hispanic women perceived health as being independent and having the ability to do the daily activities.

On reviewing the literature, only one study has focused on a sample from the Middle East using focus groups discussions. [6] Although the study focused only on men, it was found that health is meeting the economic demands of the family and the state of absence of illness. They described praying in terms of giving relaxation and mental peace.

In conclusion the majority of literature available on the meaning of health is largely guided by theoretical debate among authors themselves rather than by empirical means. Further, reported studies have tended to be small-scale research, focusing on only one gender or age group, and their conclusions were not verified by replication. [13,14,32] Given the differences in cultures, beliefs, religions and health care system, the applicability of the existing evidence to the Jordanian's 
context might be questioned. There is therefore a need for examine how hospital patients in Jordan conceptualise health.

\subsection{Study Purpose}

The study examines hospital patients' understanding of health and its cultural constructions in Jordan.

\section{Methods}

A qualitative design approach using four focus group discussions with patients was used. Health services in general need to incorporate patients' voices, which is better explored through utilising focus group discussions. [33] In particular, hospital patients may share similar experiences of nursing care and interaction amongst them in a friendly and social environment may enable a more comprehensive picture about health to emerge. [34]

\subsection{Setting}

The study was conducted in a large 500- bed hospital in Amman. The hospital provides full health services for inpatients and outpatients in a wide area comprising approximately 300,000 people. The study was performed on patients admitted to surgical and medical wards $(n=4)$. The wards were dedicated to provide care for patients undergoing general surgical and medical procedures.

\subsection{Sampling}

The first author approached patients in surgical and medical wards and selected them purposively. A purposive sample was used on the basis of availability and eligibility, with no attempt at randomization. Inclusion criteria included patients aged 18 years and older, who are physically and psychologically able to participate and spent at least one week in the hospital. This is in order to allow patients to be familiar with the surrounding environment, and experience adequate contacts with health professionals. Two focus group discussions were conducted with medical patients, including 5 women and 7 men respectively. Then, two focus group discussions were undertaken with surgical patients, including 6 women and 5 men. Therefore, the total sample included twenty three patients. The mean age was 45.8 years. Most participants were married $(n=20)$ and retired $(\mathrm{n}=17)$. Educational backgrounds varied: ten participants were illiterate, eight educated at secondary school level (Twjehi), two had a diploma degree and three were undergraduate students. The sample included diverse surgical and medical 
cases including wound debridement, appendectomy, peptic ulcer, food poisoning, and cholecystectomy.

\subsection{Data collection}

Although it is suggested that involving men and women in same group discussions could achieve a high level of comprehensiveness, this suggestion has been deemed unsuitable [35]. For cultural and religious reasons, men and women were separated. In each setting (surgical and medical wards) separate focus group discussions for men and women were arranged. Two discussions with women were undertaken by a female moderator with an MSc degree in nursing. The moderator received training on how to conduct effective focus group discussions. On the other hand, two focus group discussions were undertaken with men and moderated by the first author, who is male. All discussions were held in a comfortable and adequately ventilated room in the hospital.

Demographic information was collected before the discussions were undertaken in order to understand the characteristics of the samples. Patients were asked to express their views towards health, their own conceptualisations, and actions to keep them healthy. These questions were based on the existing literature. $[6,14$, 32] At the end of each discussion, a verbal summary with the help of the participants was produced. The mean time of the discussions was about 40 minutes. All discussions were digitally recorded.

\subsection{Ethical considerations}

Ethical approval was secured from the Research Committee at the Jordanian hospital.

All participants were assured that this research was confidential and there was no personal risk involved. In addition to the information sheet, a signed consent form was also obtained from each participant. They were assured that participation in this study was entirely voluntary and that they could withdraw at any time. Patients were assured that refusing to take part in the study would not affect in any way the quality of care provided to them. Generally, the confidentiality was assured by replacing identified names with codes.

\section{Data Analysis}

All focus group discussions were undertaken using the Arabic language. This was the participants' native language. Then, the Arabic transcript was translated into English by a bilingual professional. The authors carefully examined the transcription to ensure congruence among Arabic language and the English translation to ensure the participants' original meaning. All qualitative data collected were analysed thematically. The analysis process involved two key 
stages. Firstly, manuscripts generated by each focus group discussion were analysed separately. Secondly, all sets of data emanating from all the focus group discussions were examined in relation to a certain theme. Each manuscript was transcribed verbatim into a separate identified folder. The digitally recorded focus discussions were re-played many times to ensure adequate understanding of the data obtained.

The manuscripts were read through frequently, to become familiar with the overall picture of the data. Then, line by line a search of the manuscripts was done to scan the central themes. This included repeated ideas or statements that say something. [36] This process was accompanied by making notes about each manuscript using different colours for different themes (e.g. green- the meaning of health, red - actions to maintain health).

Once the main themes were highlighted, a category system was created for each manuscript (e.g. Category One: all themes about the meaning of health and related extracts). Careful attention was paid to obvious ambiguities, latent disagreement and "unfinished business" that arose in the course of the group discussions. [37] Finally, during analysis, data emanating from group discussions involved examples of the discursive nature of the method by reporting two or more participants in any extracts rather than focusing on an isolated excerpt offered by a certain individual.

Whilst health is a unified concept, its different aspects were divided in order to detect the level of understanding and the prevalence of a certain aspect (e.g. physical health). It was decided to categorize and present images about health according to the level of complexity. If the image has only one aspect of health to focus on, the complexity level is 1 . On the other hand, if the image involves two aspects of health, the level of complexity is 2 . Finally, if it involves two or more aspects of health and linked to each other in one response; the complexity level would be 3 .

\subsection{Trustworthiness of Qualitative Data}

The elements of trustworthiness in the current research such as credibility, dependability, and transferability were addressed. It was argued [38] that in order to enhance the credibility of qualitative data analysis, prolonged engagement with participants and their environment is needed. Prior to data collection, the current author spent two weeks in the hospital informally talking with potential participants about the research topic and related aims. Doing so could enable indepth understanding of culture and language and could aid the development of trust and rapport with participants. [39]

The credibility of the qualitative data generated was addressed by some people that checked the data. Thus, the possibility of misinterpretations of certain phrases was kept to a minimum and thus the risk of obtaining low credibility of data was diminished. 
With respect to dependability, it was achieved by inviting a research colleague to categorise independently a random selection of manuscripts. The first author and the independent researcher then discussed the agreement or disagreement of certain themes found in the manuscript. Finally, the transferability of this research was achieved by offering a sufficient descriptive account of findings and their development. [23].

\section{Findings}

\subsection{Images of health among patients}

The cross-analysis shows 3 key images or themes about health. Each image has a mixture of elements supporting its development and structure. The inductively derived analysis further shows that the participants carry out certain activities to maintain good health. Images of health and relevant clusters of meaning are explored below.

\subsubsection{Health is the freedom from illness (Complexity Level 1)}

The analysis of focus group discussions $(n=4)$ found that being free from illness is a key image of health among the participants. Although they used different descriptions, they felt that enjoying good physical health would ensure health gain and enable them to carry out daily activities. Elements of relevance to this image are "having no health problems", "taking no medicine" and "not being obese". In addition to the understanding of health elicited, data show that participants believe that certain activities are prerequisite for physical health and thus prevent the occurrence of illness (e.g. eating healthy food). As the responses were underpinned by one aspect of health, the construction of the image was simple and labelled as complexity level 1 . The image and its elements are a feature of the following extracts:

"health is having no health problems...I have 5 of them..... I am healthy when I can walk from the beginning of the corridor to its end" (Medical male patient 1).

That is right; health means feeling well and you do not need to take medicine. (Medical male patient 2).

Similar themes were found in the focus group discussion with female patients. Their responses were more concerned about the ability to work at home without experiencing the symptoms of illness. 
"The first thing comes to my mind is the freedom from illness" (medical female patient 3) "That is right" (medical female patient 4).

"Before I became ill, I used to be happy and work so hard at home and do lots of cooking and washing in the kitchen (Medical female patient 5)

Other responses were accompanied by enhancing health actions and seeking health behaviour:

"I think that health is not having diabetes. I use herbs for my health problems but if they do not work, I go to my doctor" (surgical female patient 1)

Two participants in the same group pointed out further that in order to be physically healthy, you need fresh food. The second extract indicates interestingly the link between spiritual beliefs and health.

"good food makes you healthy, eating fresh vegetables and fruits is better than fatty food. You know sometimes you find the treatment in your kitchen! (Surgical female patient 5).

"health is not being obese, you know obesity causes heart problems When I get unwell, I go to my doctor and stay at home and pray and recite Quran and then I feel less stressed" (Surgical female patient 6).

The above evidence indicates that physical health is perceived as a tool enabling participants to carry out daily activities such as walking and working at home. Thus, health is more concerned with the physical function as opposed to positive health which focuses on maximizing the potential of individual and fostering personality growth. [24] Likewise, it should be noted that physical ability was associated with two physical locations. Generally the "home" and specifically the "kitchen". Given that only female participants generated these links, they might be indicative of their gendered roles in the family as homemakers focusing on household issues.

\subsubsection{Health as having good social and psychological health (Complexity Level 2)}

The analysis reveals more complex development of the interpretations of health reported by the participants. In line with previous studies, [6, 14] viewing health from both social and psychological perspectives as an interpretation was felt by 
almost half of participants $(n=11,47 \%)$. Given that these twin aspects of health were expressed together, they were not separated in the analysis process. Being with other family members and living without stress is the feature of this image. The notion that medical health alone is not enough is also a key principle of this image. These elements are encapsulated in the following extracts:

"...health is not only good physical health: it is also concerned about psychological health. ...many problems are related to the social and psychological issues" (Surgical male patient 1)

“...yes plenty of problems are caused by psychological reasons. If you are relaxed and happy from inside your overall health will be good.praying is good for that.(surgical male patient 2)

"That is right,...You could even have cancer but if you have good socio-psychological support ...you would look like someone suffering from [nothing]... (surgical male patient 3 ).

Female patients also felt that psychological health is important for achieving your goals:

"psychological status is important. When you feel happy and have no stress, you study and work better" (medical female patient 2).

However, within the context of psychological health, a participant criticised nurses and stated that:

"as a patient, I think that nurses need to focus on psychological aspects. They care only about treatment and injections!" (surgical male patient 6)

Given the strong ties among family members in Jordan and the popularity of the extended family system, findings indicate that focusing only on medical issues might not be congruent with the patients' needs.

\subsubsection{Health as being physically, psychologically, socially and economically satisfied: (Complexity Level 3)}

The cross analysis of focus group discussions $(n=4)$ reveals that out of all participants $(\mathrm{n}=23)$, six of them $(26 \%)$ perceived health in a more systematic and complex way. Whilst minority participants offered evidence about how the holistic meaning of health can be contextually constructed. In addition to the recognition of images outlined above, it was found that good economic status is a 
contributing factor which might shape other aspects of health (e.g. physical and psychological). The image has diverse but interrelated components. Being able to work and having a post were seen as a prerequisite for good economic stability and enjoy other aspects of health such as the physical and psychological. The illumination of this economic image of health is given and its links verified by the following extracts:

"it is not only about physical and psychological health. Health is being independent economically and having a post. I wish I could go back to my work and support myself and my family.health is money!" (medical male patient 4).

A university student also expressed his frustration and anxiety due to the lack of money. According to him, this affected his recovery and psychological status.

"I feel so sad when I see my dad work so hard to offer me the cost of treatment.. if my economic status was good, I would not worry about this and psychologically I would feel satisfied and sleep well!!" (Medical male patient 5).

"That is right, health means to have money so you do not start begging to cover the cost of your expenses! This alone makes you socially isolated and psychologically worried" (Medical male patient 3 ).

As highlighted above, participants felt that being able to meet the economic demands of the whole family is an essential element of good health. Whilst other aspects of health were acknowledged, it seems that being economically satisfied is at the heart of their responses. These findings are indicative of recognition of the overlapping issues related to health. Some phrases used by participants such as "socially isolated" and "psychologically worried" shed light on the impact of the economic burden on self growth. These elements are against the thesis of selfempowerment ideology and thus heath promotion. [40-41]

In addition to the lack of support at a national level (e.g. free treatment), the extended family as a support and burden system might add further economic constraints on the shoulder of patients. That is, it seems that there are broader issues interplaying with patients' health at both individual and national levels.

\section{Discussion}

Using focus group discussions, the study examined hospital patients' understanding of health in Jordan. Whilst health is diverse, the study findings resonate with previous research and highlight the importance of the medical view of health among people in general and in particular patients in hospital. [6, 13,14] 
Although other aspects of health are recognised by participants, being physically healthy was the focus of their conceptualizations. Within the hospital context, this understanding of health might be explained by a number of factors. As all participants had medical problems and were admitted for treatment, it might be possible that their first concern was to regain physical health and go home. The focus on the medical view of health may also be associated with the environment itself. [7, 28] During the time in hospital, many patients are often exposed to medical treatment, investigations and health stories from other patients.

Interesting findings to note are the link between health and religious actions. This is manifested by praying and reciting Quran. These have been seen by patients as "stress busters". Earlier work showed that religious involvement in care is correlated with decreased morbidity and mortality. $[42,43]$ is associated with up to 7 years longer life expectancy, [29] had a beneficial impact on blood pressure management, [44] and might help patients to adjust to socio-economic problems. [45] Whilst the study aim is not to check the link between a cause and an effect, using the "five Rs of spiritual care" might be a useful tool to explore patients" needs of spiritual care and its impact on their health. [46] This includes, Reason (the purpose of life), Reflection (reflect on the experience of illness), Religion (the framework in which individuals express their spirituality), and restoration, (the way spirituality affects health). Although these elements need to be examined fully from different faith perspectives, they could offer a foundation to explore the spiritual needs of patients.

Nevertheless, a word of caution must be sounded here. Whilst spiritual care is needed, patients should not be exclusively encouraged to focus on religious belief and ignore medical treatment. This could lead them to think that inadequate faith is the cause of their illness [47] and thus to minimise their adherence to the prescribed treatment plan. It is crucial therefore those health care providers offer a balanced approach, medically oriented interventions and informed by spiritual care. By incorporating what patients' believe about health into the framework of care, a high quality of life can be achieved together with treatment compliance. [6, $14,48]$

Previous studies have offered important insights into the meanings of health, but they focussed on one gender. [13, 31] In this study, whilst similarities in understanding health are acknowledged among men and women (e.g. health as the absence of illness), there are some gender associated differences and thus potential implications for practice. Men in this study were more concerned about financial status than women. Although some evidence suggests that women were concerned about eating healthy food, no explicit references were made to financial burdens and health.

By contrast, the perception of health among men revolved around having employment and adequate money in order to meet the financial commitments of their families. This highlights the fact there is no self-actualisation if the basic needs are not met (e.g. treatment). That is, in the current analysis, patients might not be able to reach the state of actualisation unless they are financially well 
supported. [49] It is worth noting that the average age of male participants in both group discussions was 45 years. This might be associated with having more economic problems due to increasing demands such as raising children and paying for their education. Indeed, in Jordan men are often responsible not only for offering financial support to their own families but also to their parents, sisters and unemployed brothers. Specifically, men in this study might have felt more concerned about economic issues given their role as breadwinners in Jordanian society.

Likewise, from the women's perspective, ironically it should be noted that physical ability was associated with two physical locations, the "home" and specifically the "kitchen". Given that only female participants identified these links, this might be indicative of their gendered roles in the family as homemakers focusing on household issues. In other words, as validated by a previous Jordanian study [50] and Hispanic research, [14] feeling physically unwell might compromise the fulfilment of such roles and thus affect the overall health of the family.

\subsection{Implication for Practice}

The main findings of this study showed that the role of women and men in Jordan shapes their understanding of health. Thus, it seems that there is a danger of utilising a standardised caring approach which may conflict with gender issues and therefore health promotion needs during hospitalisation. Previously it was found that the majority of female patients prefer to be looked after by female nurses. [51] The current study however reveals that the issue is not only about gender preferences but also about the differences in perceptions and thus expectations. Such differences are of importance to the development of delivering health promotion activities sensitive to cultural and gender issues. This is because the future of health care depends not only on scientifically based evidence but also on recognition of population diversity and people's cultural understanding of health itself. [17, 20,52] As a result, hospital nurses should incorporate patients' constructs of health into the framework of the patient's daily care plan and health promotion activities.

\subsection{Study strengths and limitations}

This is, to date, one of a few studies that try to understand hospital patients' conceptualisations and cultural constructs of health. There are however some limitations to keep in mind. Caution must be applied and considerations must be given to the methods and study design. Mainly, qualitative research has been criticized on the ground of ignoring issues of validity and reliability and for being anecdotal. [53] The reliability of this work was enhanced by respondents' checking of findings (trustworthiness of data). The findings however might be considered as a benchmark against which further research in this area is developed 
and thus sharpen the generalisability of this work. Future research using a quantitative approach with a larger sample size is essential to replicate and expand upon the relevancy and accuracy of the themes that emerged in this study.

\section{Conclusion}

In confirmation of previous research, qualitative data from this study showed some complex images and cultural constructs of health among patients. These images are shaped by the gender of patients. Hospital nurses are urged to incorporate them into the framework of the patient's daily care plan to achieve a higher level of their self actualisation and thus compliance with the prescribed treatment. Failure to do so might result in a less informed and less likely successful health promotion strategies.

\section{References}

[1] Catford, J., The Bangkok Conference: steering countries to build national capacity for health promotion. Health Promotion International, 2005. 20(1): 1-6.

[2] Mittelmark, M.B., Setting an ethical agenda for health promotion. Health Promotion International, 2008. 23(1): 78-85.

[3] Pelikan, J.M., K. Krajic, and C. Dietscher, The health promoting hospital (HPH): concept and development. Patient Education and Counseling, 2001. 45(4): 239-243.

[4] Seedhouse, D., Health promotion: Philosophy, Prejudice and Practice, 2nd ed John Wiley, West Sussex, 2004.

[5] Shoqirat, N., The role of jordanan hospital nurses in promoting patients health (PhD thesis). Queen Margaret University, Edinburgh, 2009.

[6] Hjelm, K.G., et al., Beliefs about health and diabetes in men of different ethnic origin. Journal of Advanced Nursing, 2005. 50(1): 47-59.

[7] Tones, K. and J. Green, Health Promotion: Planning and Strategies. Sage Publications, London, 2004.

[8] Casey, D., Nurses' perceptions, understanding and experiences of health promotion. Journal of Clinical nursing, 2007. 16(6): 1039-1049.

[9] Cross, R., Accident and emergency nurses' attitudes towards health promotion. Journal of Advanced Nursing, 2005. 51(5): 474-483.

[10] Whitehead, D., et al., Health promotion and health education practice: nurses' perceptions. Journal of Advanced Nursing, 2008. 61(2):181-187.

[11] Irvine, F., Exploring district nursing competencies in health promotion: the use of the Delphi technique. Journal of Clinical Nursing, 2005. 14(8): 965975. 
[12] Piper, S., Health Promotion for Nurses: Theory and Practice. Routledge, Abingdon, 2009.

[13] Maddox, M., Older women and the meaning of health. Journal of Gerontological Nursing, 1999. 25(12):26-32.

[14] Yoho, M.J. and I. Ezeobele, Health and meaning: A perspective of Geriatric Hispanic Women. Geriatric Nursing, 2002. 23(5): 268-271.

[15] Gharaibeh, M., R. Al-Ma'aitah, and N. Al Jada, Lifestyle practices of Jordanian pregnant wmen. International Nursing Review, 2005. 52(2): 92100.

[16] Shoqirat, N. and S. Cameron, Promoting Hospital Patients' Health in Jordan: Rhetoric and Reality of Nurses' Roles. International Journal of Nursing 2012. 1(1): 28-37.

[17] McLennan, V. and F. Khavarpour, Culturally appropriate health promotion: its meaning and application in Aboriginal communities. Health Promotion Journal of Australia, 2004. 15(3): 237-242.

[18] Bowling, A., Measuring Health: A review of Quality of Life Measurement Scales. Open University Press, England, 2004.

[19] Higgins, P.G. and C.D. Learn, Health practices of adult Hispanic women. Journal of Advanced Nursing, 1999. 29(5): 1105-1112.

[20] Kim-Godwin, Y.S., P.N. Clarke, and L. Barton, A model for the delivery of culturally competent community care. Journal of Advanced Nursing, 2001. 35(6):918-925.

[21] Ogden, J., et al., What is health? Where GPs' and patients' worlds collide. Patient Education and Counseling, 2001. 45(4): 265-269.

[22] Buchanan, D.R., Perspective: A new ethic for health promotion: Reflections on a philosophy of health education for the 21 st century. Health Education \& Behavior, 2006. 33(3): 290-304.

[23] McBride, A., Health promotion in the acute hospital setting: the receptivity of adult in-patients. Patient Education and Counseling, 2004. 54(1): 73-78.

[24] De Chavez, A.C., et al., Understanding and researching wellbeing: Its usage in different disciplines and potential for health research and health promotion. Health Education Journal, 2005. 64(1): 70-87.

[25] WHO, Constitution of the WHO. Reprinted in: Basic documents. WHO, Geneva, 1946.

[26] King, I.M., King's conceptual framework and theory of goal attainment. National League for Nursing, New York, 1990.

[27] Ewles, L. and I. Simnett, Promoting Health: A practical Guide., ed. 5th. Bailliere Tindall, Toronto,2003.

[28] Pender, N.J., C.L. Murdaugh, and M.A. Parsons, Health Promotion in Nursing Practice. 5th ed. Upper saddle river, lavoisier, 2002.

[29] Helman, C.G., Culture, health and illness. Hodder Headline Group, Arnold, 2001.

[30] Tones, K. and S. Tilford, Health promotion: effectiveness, efficiency, and equity. Nelson Thornes, Cheltenham, 2001. 
[31] Dickinson, R. and A. Bhatt, Ethnicity, health and control: results from an exploratory study of ethnic minority communities' attitudes to health. Health Education Journal, 1994. 53(4): 421-429.

[32] Paxton, S., A. Sculthorpe, and K. Gibbons, Concepts of health in Australian men: a qualitative study. Health Education Journal, 1994. 53(4): 430-438.

[33] Umaña-Taylor, A.J. and M.Y. Bámaca, Conducting Focus Groups with Latino Populations: Lessons from the Field. Family Relations, 2004. 53(3): 261-272.

[34] McKinley, R.K., et al., Reliability and validity of a new measure of patient satisfaction with out of hours primary medical care in the United Kingdom: development of a patient questionnaire. BMJ, 1997. 314(7075): 193-198.

[35] Sim, J., Collecting and analysing qualitative data: issues raised by the focus group. Journal of Advanced Nursing, 1998. 28(2): 345-352.

[36] Burnard, P., A method of analysing interview transcripts in qualitative research. Nurse Education Today, 1991. 11(6): 461-466.

[37] Kitzinger, J. and R.S. Barbour, Developing Focus Group Research: Politics, Theory and Practice. London: Sage Publications Ltd, 1999.

[38] Polit, D.B. and C. Hungler, C. Essentials of nursing research: Methods, Appraisal, and Utilization, Lippincott Williams \& Wilkins, Philadelphia 2006.

[39] Gillis, A. and W. Jackson, Research for Nurses: Methods \& Interpretation. F.A Davis Company, Philadelphia ,2001

[40] Mok, E. and B. Au- Yeung, Relationship between organizational climate and empowerment of nurses in Hong Kong. Journal of Nursing Management, 2002. 10(3): 129-137.

[41] Houston, A.M. and S. Cowley, An empowerment approach to needs assessment in health visiting practice. Journal of Clinical Nursing, 2002. 11(5): 640-650.

[42] Matthews, D.A., et al., Religious commitment and health status: a review of the research and implications for family medicine. Archives of Family Medicine, 1998. 7(2): 118-123.

[43] Oman, P.D., D., et al., Religious attendance and cause of death over 31 years. The International Journal of Psychiatry in Medicine, 2002. 32(1): 69-69.

[44] Walsh, A., Religion and hypertension: testing alternative explanations among immigrants. Behavioral Medicine, 1998. 24(3): p. 122-130.

[45] Van Poppel, F., J. Schellekens, and A.C. Liefbroer, Religious differentials in infant and child mortality in Holland, 1855-1912. Population Studies, 2002. 56(3): 277-289.

[46] Govier, I., Spiritual care in nursing: a systematic approach. Nursing Standard 2000. 14(17):32.

[47] Sloan, R.P. and E. Bagiella, Claims about religious involvement and health outcomes. Annals of Behavioral Medicine, 2002. 24(1): 14-21.

[48] Lo, B., et al., Discussing religious and spiritual issues at the end of life. Journal of the American Medical Association, 2002. 287(6): 749-754. 
[49] Rafael, F. and R. Adeline, The politics of health promotion: Influences on public health promoting nursing practice in Ontario, Canada from Nightingale to the nineties. Advances in Nursing Science, 1999. 22(1): 2327.

[50] Mahasneh, S.M., Health perceptions and health behaviours of poor urban Jordanian women. Journal of Advanced Nursing, 2001. 36(1): 58-68.

[51] Ahmad, M.M. and J.A. Alasad, Patients' preferences for nurses' gender in Jordan. International Journal of Nursing Practice, 2007. 13(4): p. 237-242.

[52] Jones, K.E., C.R. Brockway, and R.E. Atkinson, Survey of health promotion organisational arrangements and levels of service for health promotion. Public Health, 1995. 109(1): 31-39.

[53] Glaser, B.G. and A.L. Strauss, The Discovery of Grounded Theory: Strategies for Qualitative Research. De Gruyte, New York, 1967. 